\title{
A Novel Guide-Wire Technique for Repositioning a Nasobiliary Catheter from Mouth to Nostril without Using a Nelaton Tube
}

\author{
Tetsuya Hamano $^{a}$ Shuhei Yoshida ${ }^{b}$ Takayoshi Nishino $^{b}$ Junichi Akao ${ }^{b}$ \\ Izumi Shirato $^{\text {b }}$ Tomoko Tagata $^{a}$ Yuichi Ikarashi ${ }^{b}$ Yutaka Mitsunaga ${ }^{b}$ \\ Masahiko Shimadab Atsushi Mitsunaga ${ }^{a}$ \\ Departments of a Endoscopy and ${ }^{b}$ Gastroenterology, Yachiyo Medical Center, Tokyo Women's Medical University, \\ Chiba, Japan
}

\section{Key Words}

Guide wire · Nelaton tube - Endoscopic nasobiliary drainage . Endoscopic retrograde cholangiopancreatography

\begin{abstract}
Objective: We aimed to assess the usefulness of a novel guide-wire technique for repositioning without the use of a Nelaton tube and to compare this to the conventional technique. Subjects and Methods: A total of 50 patients who underwent endoscopic nasobiliary drainage (ENBD) at the Yachiyo Medical Center, Chiba, Japan, were enrolled into the study. The patients were randomly divided into 2 groups according to the use of a novel guide-wire technique $(n=28)$ or the conventional technique $(n=22)$. The ENBD catheters were repositioned from the mouth to the nose. The primary end point was the procedural time from the insertion of the Nelaton tube or guide wire into the nostril until the ENBD catheter had been repositioned in the nose. The secondary end point was the success rate of the procedure. Results: The mean procedure time of our technique (120.8 s) was shorter than the traditional technique (131.9 s), but this difference was not statistically significant $(p=0.56)$. Our technique did not involve the use of the Nelaton tube, and so could save the cost of USD 1.17 per patient. The novel technique did not
\end{abstract}

require the removal of the mouthpiece with a laryngoscope or the use of a Nelaton tube, and no postural change was necessary. A single operator performed the novel procedure unassisted. No adverse events were observed relating to either the novel or the traditional technique. Conclusions: The novel guide-wire technique for repositioning ENBD catheters was effective and is recommended for use.

(c) 2015 S. Karger AG, Basel

\section{Introduction}

Endoscopic nasobiliary drainage (ENBD) catheters are known to be very useful and effective devices for temporary biliary drainage in acute suppurative cholangitis [1]. ENBD catheters have been a standard treatment strategy for drainage of the biliary system in patients with cholangitis for more than 30 years [1]. They are most often used in patients with a malignant stricture secondary to a pancreaticobiliary cancer as well as in patients with stones in the common bile duct $[2,3]$. However, the conventional technique has the following critical disadvantages. (1) The operator cannot perform the procedure unassisted, and the mouthpiece must be removed from a sedated patient with a change of position. (2) The opera-

\begin{tabular}{ll}
\hline KARGER 125:s & $\begin{array}{l}\text { () 2015 S. Karger AG, Basel } \\
1011-7571 / 15 / 0244-0339 \$ 39.50 / 0 \quad \text { Karger }\end{array}$ \\
$\begin{array}{l}\text { E-Mail karger@karger.com } \\
\text { www.karger.com/mpp }\end{array}$ & $\begin{array}{l}\text { This is an Open Access article licensed under the terms of the } \\
\text { Creative Commons Attribution-NonCommercial 3.0 Un- } \\
\text { ported license (CC BY-NC) (www.karger.com/OA-license), } \\
\text { applicable to the online version of the article only. Distribu- } \\
\text { tion permitted for non-commercial purposes only. }\end{array}$
\end{tabular}

Takayoshi Nishino, MD

Yachiyo Medical Center, Tokyo Women's Medical University 477-96 Owada-Shinden

Yachiyo, Chiba 276-8542 (Japan)

E-Mail takanishino@mm.em-net.ne.jp 
Table 1. Clinical characteristics of all ENBD patients

\begin{tabular}{lclll}
\hline Characteristics & $\begin{array}{l}\text { Whole } \\
\text { cohort } \\
(\mathrm{n}=50)\end{array}$ & $\begin{array}{l}\text { Conventional } \\
\text { technique } \\
(\mathrm{n}=22)\end{array}$ & $\begin{array}{l}\text { Guide-wire } \\
\text { technique } \\
(\mathrm{n}=28)\end{array}$ & $\begin{array}{l}\mathrm{p} \\
\text { value }\end{array}$ \\
\hline Age, years & $70.06 \pm 12.12$ & $70.50 \pm 13.30$ & $69.71 \pm 11.34$ & 0.82 \\
Gender, \% & 42 & 40.9 & 42.9 & 0.89 \\
$\quad$ Female & 58 & 59.1 & 57.1 & \\
$\quad$ Male & 96 & 95.5 & 96.4 & 0.86 \\
Success rate, \% & $125.7 \pm 64.02$ & $131.9 \pm 71.64$ & $120.8 \pm 58.34$ & 0.56 \\
Procedure time, $\mathrm{s}$ & & & & \\
\hline
\end{tabular}

tor must look into the patient's oropharynx and draw the end of the Nelaton tube out through the patient's mouth, and thus the procedure is very uncomfortable for the operator as well as the patient. Japanese endoscopists have tried to overcome these disadvantages for many years. Although a simpler, guide-wire technique that does not involve the use of a Nelaton tube was presented for the first time at the 48th Annual Meeting of the Japan Biliary Association in 2012 [4], no literature on this technique has been published yet. In this study, we evaluated the guidewire technique in comparison to the traditional technique.

\section{Subjects and Methods}

This was a prospective, single-blind, controlled pilot study of the novel guide-wire technique for repositioning ENBD catheters from the mouth to the nose, and it was conducted at a single center. The study subjects were 50 consecutive patients ( 28 men and 22 women) who underwent ENBD at the Yachiyo Medical Center, Tokyo Women's Medical University (Chiba, Japan) between September 2012 and June 2013. The clinical characteristics of the patients are shown in table 1. Informed consent was obtained from all patients before endoscopic retrograde cholangiopancreatography (ERCP) was performed. The study was performed in accordance with the ethical standards of the institutional review board and the Declaration of Helsinki (protocol 139428, 1 August 2012). Midazolam $(5 \mathrm{mg})$ and pentazocine $(7.5 \mathrm{mg})$ were intravenously injected for the induction of sedation and analgesia, and supplemental doses were administered as needed. Stenting of the ENBD tube under fluoroscopic guidance was performed in all patients with a duodenoscope (JF-260V; Olympus, Tokyo, Japan). The ENBD tube used in all cases was a 6-Fr reverse alpha type (Flexi$\mathrm{ma}^{\mathrm{TM}}$ Nasobiliary Catheter Single Pigtail, Cat. No. 4012, Boston Scientific, Tokyo, Japan), and a Nelaton tube was used (Angel Robinson catheter A, size 6, Cat. No. 130202, Kosan Co., Tokyo, Japan) or a guide wire (the 0.035 -inch Hydra Jagwire ${ }^{\mathrm{TM}}$ or the 0.025 -inch VisiGlide $^{\mathrm{TM}}$, Boston Scientific, Tokyo, Japan). Procedural time, age, gender and success rate (\%) were evaluated and compared with the conventional technique. The two techniques were randomly performed by two endoscopists (T.H. and J.A.), each of whom had experience in performing ERCP in $>1,000$ cases. The patients were blinded to the randomization sequence. They were randomized in advance (by means of randomization software) into the novel technique and traditional technique groups and then distributed by a third person to the endoscopic center using opaque sealed envelopes. They were randomized before the endoscopists evaluated their clinical parameters. The primary end point was the procedural time. Procedural time was measured from the moment of insertion of the Nelaton tube or guide wire into the nostril until the ENBD catheter had been repositioned in the nose. Procedural time was evaluated only in patients in whom the ENBD catheter had been successfully repositioned. The cost of each procedure was also assessed. The secondary end point was the success rate of the procedures.

\section{The Novel Guide-Wire Technique}

The novel technique only required the use of a guide wire that is commonly used in ERCP. One end of the guide wire was held straight with the operator's right hand. With the operator's left hand, a loop was made in the middle portion of the guide wire, and this loop was then held at its base where the wire crossed (fig. 1a). With the patient under sedation in the abdominal position, the straight end of the guide wire was passed directly through a nostril into the nasopharynx. The operator inserted the loop through the mouthpiece and advanced it to the oropharynx. Under X-ray fluoroscope guidance, the straight end of the guide wire was caught in the loop in the hypopharynx and led out of the mouth (fig. 1b). Finally, the ENBD catheter was drawn from the pharynx out through the nostril with the guide wire in a retrograde fashion (fig. 1c, d). The guide wire caught in the loop was easily visible under fluoroscopy (fig. 2). It is noteworthy that no laryngoscope, Nelaton tube or postural change was used in this novel technique. Physical assistance by other people is required for the conventional technique, but a single operator performed the procedure unassisted for the guide-wire technique.

\section{The Conventional Technique of Repositioning the ENBD}

\section{Catheter}

This procedure for the repositioning ENBD tube from the mouth to the nostril has been described previously [5]. Briefly, after insertion of the ENBD tube transorally, a Nelaton tube is inserted transnasally in order to engage the ENBD tube with a postural change. The mouthpiece is then removed and the leading end of the Nelaton tube is orally pulled out using surgical forceps under the guidance of a laryngoscopy. The tubes are connected by inserting the end of the ENBD tube into the Nelaton tube. Finally, the Nelaton tube and the connected ENBD tube are nasally pulled back out.

\section{Evaluation of the Method}

Data are expressed as means \pm standard deviation, and the statistical analysis was performed with the Microsoft Excel (Microsoft, Redmond, Wash., USA) and GraphPad Prism v5.00 (GraphPad Software, San Diego, Calif., USA) software programs. All comparisons of parameters between the 2 groups were performed using an unpaired Student $t$ test followed by a nonparametric test. Differences between groups were considered significant when the $\mathrm{p}$ value was $<0.05$. 
Fig. 1. a-d Procedure with the novel guidewire technique to reposition the proximal end of an ENBD catheter from the mouth to the nose.
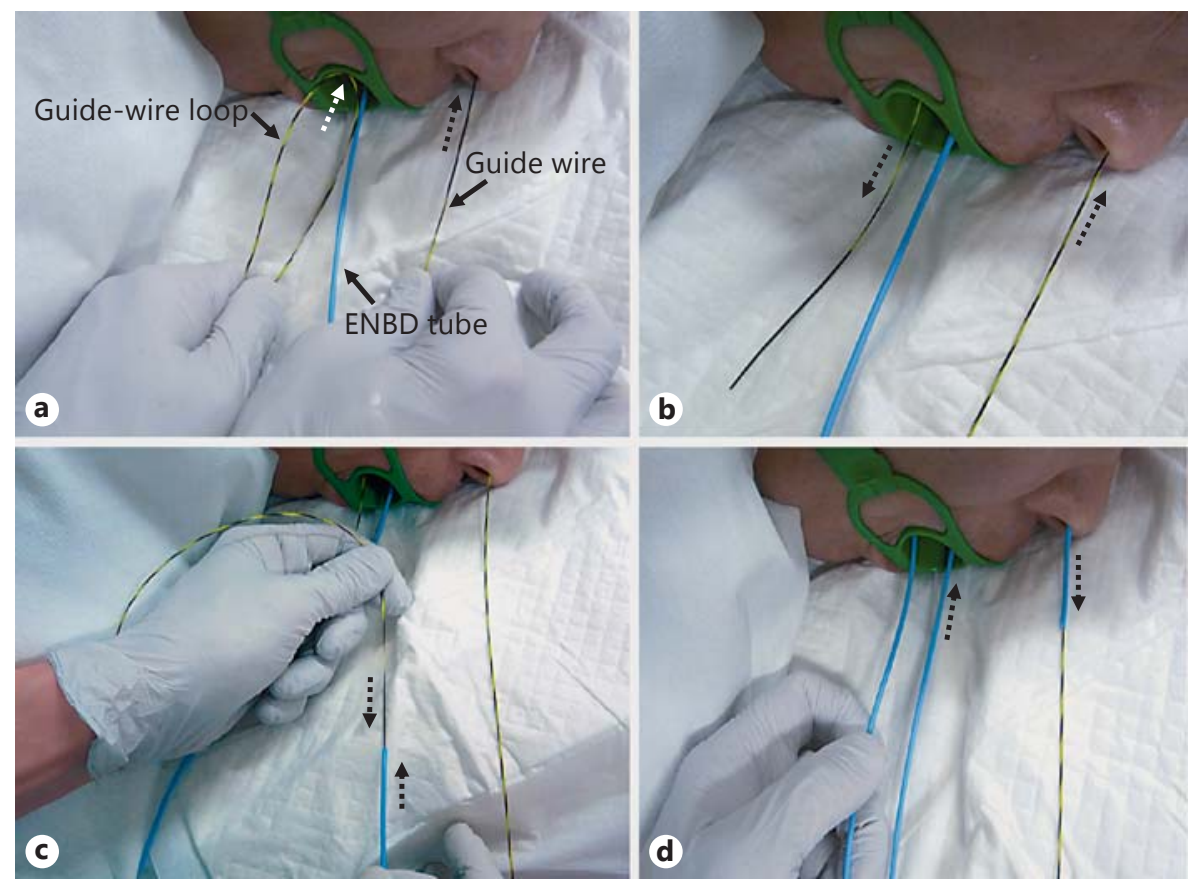

\section{Results}

There were no significant differences between the guide-wire group $(69.71 \pm 11.34)$ and the conventional group $(70.50 \pm 13.30)$ in age $(\mathrm{p}=0.82)$ or gender $(58 \%$ males and $42 \%$ females; $\mathrm{p}=0.89)$. There was a success rate for the repositioning procedure of $96.4 \%(27 / 28)$ in the guide-wire group and $95.5 \%(21 / 22)$ in the conventional group $(\mathrm{p}=0.86)$. Procedure time in the successful cases was $120.8 \pm 58.34 \mathrm{~s}$ in the guide-wire group (median 102) and $131.9 \pm 71.64 \mathrm{~s}$ in the conventional group (median $110)$, also with no statistical difference ( $\mathrm{p}=0.56$; table 1$)$. There were two repositioning failures, one caused by the inability to insert the guide-wire into the pharynx and the other by dislocation of the ENBD catheter from the bile duct during the procedure. No switches from an operator to a senior physician were required in the study. X-ray fluoroscopy during the guide-wire technique required an extra exposure time (median $70 \mathrm{~s}$ ) but the conventional technique did not. Complicated procedures in the conventional technique included removal of the mouthpiece, a postural change, laryngoscopy and the use of a Nelaton tube; these were not required in the novel technique. No patients had any adverse events related to the novel or the traditional technique. The cost of a Nelaton tube (USD 1.17 or JPY 140) was saved in the novel guide-wire technique.

A Novel Technique for Repositioning a

Nasobiliary Catheter during ERCP

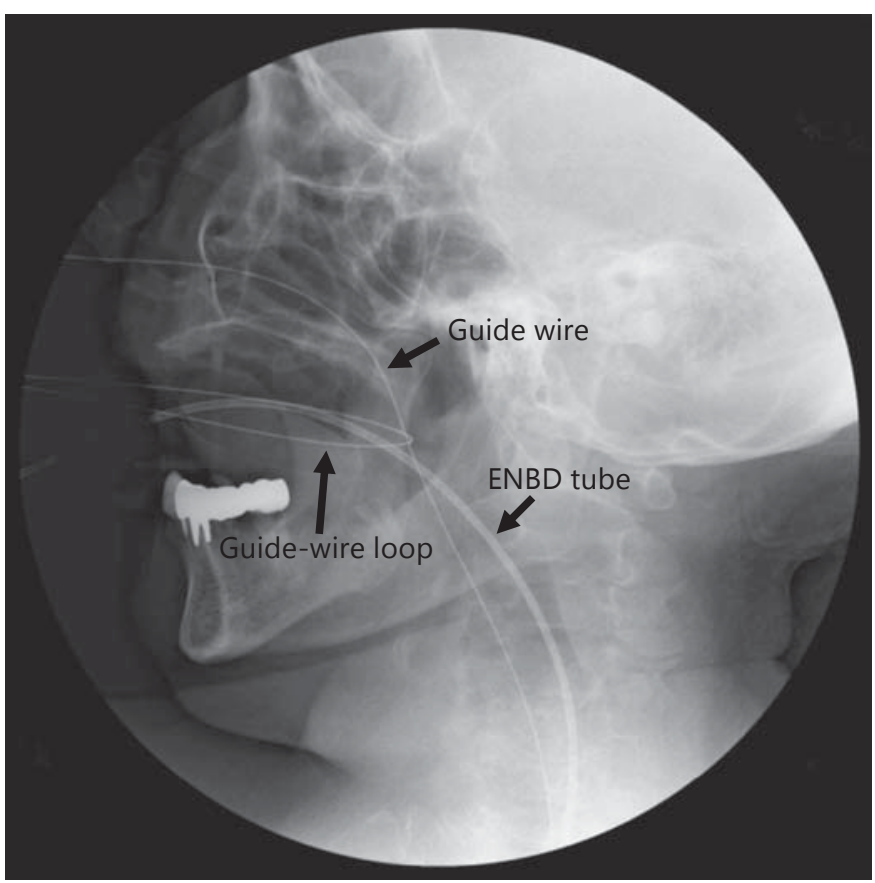

Fig. 2. Fluoroscopic view of a guide wire. 


\section{Discussion}

In this study, the guide-wire technique was a useful procedure for repositioning the ENBD catheter through the nose. This method was found to be superior in terms of feasibility, safety and visibility, and it allowed placement in a short time with X-ray fluoroscopy assistance. The device used in this novel technique was simple, available, inexpensive and uninvasive. An ENBD kit contains a guide wire, so no extra preparations were required. A Nelaton tube was unnecessary for this technique. Furthermore, all endoscopists can easily perform this technique after practising a few times because of the simpleness of the technique. There is thus a definite possibility that this novel guide-wire technique could become the first choice for ENBD catheter replacement. A Japanese group developed a 'roping technique' in 2012 [6]. Since this technique does not require removal of the mouthpiece or the use of a laryngoscope to insert the ENBD catheter into the pharynx with postural change, it seemed to be a breakthrough after the conventional technique. However, it still required painful insertion of a Nelaton tube through the nostril into the pharynx during the procedures. Another disadvantage is that the Nelaton tube is very hard to detect during fluoroscopy. Our technique, on the other hand, does not require a Nelaton tube or removal of the mouthpiece or laryngoscopy or a postural change. In addition, only a single guide wire, readily visible under X-ray fluoroscopy, is required to reposition the ENBD catheter. An interesting magnet technique that does not require removal of the mouthpiece or laryngoscopy was recently reported [7], but the use of the magnet and another suction tube involves additional costs and preparation. Furthermore, insertion of a thick Nelaton tube remains uncomfortable for patients, and the magnet method cannot be used in patients with pacemakers because the magnet can affect pacemaker function as in magnetic resonance imaging examinations. In the study reporting the magnet method, the authors themselves pointed out the problem of detachment of the magnet from the tube [8]. This indicates that magnetic attraction during this procedure can be very unstable. Furthermore, their report was not a comparative study.

Statistical comparison is essential to determine the exact benefits of a novel technique. In our study, we compared the novel guide-wire technique with the traditional technique. It is noteworthy that our technique can be used in patients with pacemakers. Our guide-wire technique is simple and noninvasive. Although guide wires are very useful tools for performing cholangiography $[8$,
9] and angiography [10] as well as for the treatment of gut obstructions [11], they are disposable products intended for only a single use. Here, we showed a use for guide wire other than cannulation during ERCP. No additional devices and costs are needed for inserting an ENBD catheter into a nostril. No adverse events were observed. The only drawback of our novel procedure was the additional 70-second X-ray exposure, but we do not consider that this has a long-term effect on patients' health. The mean procedure time of our technique was shorter than the traditional technique, although the difference did not reach statistical significance $(\mathrm{p}=0.56$; table 1$)$.

Our study had two limitations. The first was that the number of subjects was relatively small, and the second was that it was a single-center pilot study. Larger studies or multicenter clinical trials will be needed to confirm the usefulness of our novel technique.

\section{Conclusion}

The guide-wire technique for repositioning an ENBD catheter was equally effective and required only one operator. Furthermore, no adverse events were observed in the procedure. Therefore, the use of this novel technique for repositioning ENBD catheter instead of the traditional technique is highly recommended.

\section{Acknowledgment}

This study was supported in part by the Japanese Grant-in-Aid for Scientific Research, KAKENHI (grant No. 15K09027) to S.Y.

\section{Disclosure Statement}

There are no conflicts of interest.

References

$$
\begin{aligned}
& \text { Nagai N, Toli F, Oi I, et al: Continuous endo- } \\
& \text { scopic pancreatocholedochal catheterization. } \\
& \text { Gastrointest Endosc 1976;23:78-81. } \\
& 2 \text { Ogawa M, Kawaguchi Y, Tsuda S, et al: PO- } \\
& 126 \text { (a poster presentation) at the 48th Annual } \\
& \text { Meeting of the Japan Biliary Association (in } \\
& \text { Japanese). J Jpn Biliary Assoc 2012;26:523. } \\
& 3 \text { Arakura N, Takayama M, Ozaki Y, et al: Ef- } \\
& \text { ficacy of preoperative endoscopic nasobiliary } \\
& \text { drainage for hilar cholangiocarcinoma. J } \\
& \text { Hepatobiliary Pancreat Surg 2009;16:473- } \\
& \text { 477. }
\end{aligned}
$$

Hamano et al. 
4 Xu XD, Dai JJ, Qian JQ, et al: Nasobiliary drainage after endoscopic papillary balloon dilation may prevent postoperative pancreatitis. World J Gastroenterol 2015;21:24432449.

5 Tsuyuguchi T, Takada T, Kawarada Y, et al: Techniques of biliary drainage for acute cholangitis: Tokyo guidelines. J Hepatobiliary Pancreat Surg 2007;14:35-45.

6 Kunishi Y, Kaneko T, Sugimori K: Introduction of roping techniques: a new induction method of ENBD tube to nostril using a guide wire (in Japanese). Gastroenterol Endosc 2012;54:2046-2047.

7 Watanabe S, Sato T, Kato S, et al: Positioning of nasobiliary tube using magnet-loaded catheters. Endoscopy 2013;45:835-837.

$>8$ Fujita N, Sugawara T, Noda Y, et al: Snareover-the-wire technique for safe exchange of a stent following endosonography-guided biliary drainage. Dig Endosc 2009;21:48-52.

9 Herreros de Tejada A, Calleja JL, Diaz G, et al: Double-guidewire technique for difficult bile duct cannulation: a multicenter randomized, controlled trial. Gastrointest Endosc 2009;70: 700-709.

10 Takeuchi Y, Arai Y, Kasahara T, et al: Technical aspects of venous stenting in high-grade stenoses using a long guidewire between dual venous access sites. Eur Radiol 2000;10:167169.

1 Lamazza A, Fiori E, Schillaci A, et al: A new technique for placement of a self-expanding metallic stent (SEMS) in patients with colon rectal obstruction: a prospective study of 43 patients. Surg Endosc 2013;27:1045-1048. 